Supplementary Information

\title{
The Laser-Induced Potential-Jump: a Method for Rapid Electron Injection into Oxidoreductase Enzymes
}

Monica L. K. Sanchez, ${ }^{+}$Sara E. Konecny, Sarah Narehood ${ }^{+}$, Edward Reijerse, ${ }^{\ddagger}$ Wolfgang Lubitz, ${ }^{\ddagger}$ James A. Birrell, ${ }^{* *}$ R. Brian Dyer ${ }^{* *}$

AUTHOR ADDRESS

${ }^{\dagger}$ Department of Chemistry, Emory University, Atlanta, Georgia 30030, United States

${ }^{\ddagger}$ Max Planck Institute for Chemical Energy Conversion, Stiftstrasse 34-36, 45470 Mülheim an der Ruhr, Germany

$\begin{array}{ll}\text { Section 1. Materials Characterization } & \text { S2 }\end{array}$

A) Fig. S1: TEM Image of CdSe/CdS NRs $\quad$ S2

B) Fig. S2: UV-Vis Absorbance and Fluorescence Spectra of NRs $\quad$ S2

Section 2. Quantum Efficiency of Photo-driven Mediator Reduction $\quad$ S3

A) Fig. S3: Representative UV-Vis Spectra of Mediator Radicals: DQ03, DQ53 and DQ43 S3

B) Table S1: pH Dependence of QE (\%) of Photo-Driven Mediator Reduction S3

C) Table S2: Mediator Concentration Dependence of QE (\%) of Photo-Driven Mediator S3 Reduction

D) Example Quantum Efficiency Calculation $\quad \mathrm{S} 4$

Section 3. Steady-State Solution Potential during Hydrogen Production $\quad$ S5

A) Fig. S4: Steady-State Solution Potential during $\mathrm{H}_{2}$ Production and $\mathrm{H}_{2}$ produced in the $\quad \mathrm{S} 5$ absence of the enzyme

Section 4. Table S3: Fit Coefficients for TCSPC data $\quad S 6$

$\begin{array}{ll}\text { Section 5. Data Analysis Procedure for Transient Visible Measurements } & \text { S7 }\end{array}$

$\begin{array}{ll}\text { Section 6. Fitting Procedure for Time-Resolved Visible Data } & \text { S8 }\end{array}$

A) $1 \mathrm{mM}$ Enzyme Sample, TR-Vis Data $\quad$ S8

B) 4 mM Enzyme Sample, TR-Vis Data $\quad$ S8

Section 7. Calculation of Laser Induced Potential Jump $\quad$ S9

Section 8. Table S4: Summary of Solution Potential Jump Data $\quad$ S10

Section 9. Fit Coefficients for Time-Resolved IR Measurements $\quad$ S11

Section 10. Fit Coefficients for Time-Resolved Vis Measurements $\quad$ S11

Section 11. Proposed Catalytic Cycle of [FeFe] Hydrogenase $\quad S 12$

$\begin{array}{ll}\text { Section 12. References } & \text { S13 }\end{array}$ 


\section{Section 1. Materials Characterization}

\section{A) Transmission Electron Microscopy (TEM) Image of CdSe/CdS Nanomaterials}

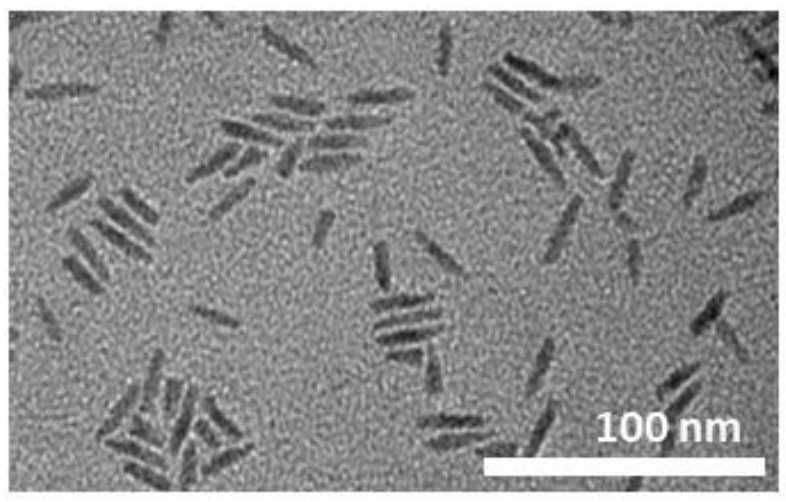

Figure S1. TEM Images of CdSe/CdS NRs. Representative TEM images of CdSe/CdS nanorods collected on a Hitachi 770 microscope with an acceleration voltage of 80 kV. Samples were prepared by evaporation on a Cu mesh grid (Electron Microscopy Sciences.)

B) Fluorescence and UV-Vis of CdSe/CdS Nanorods

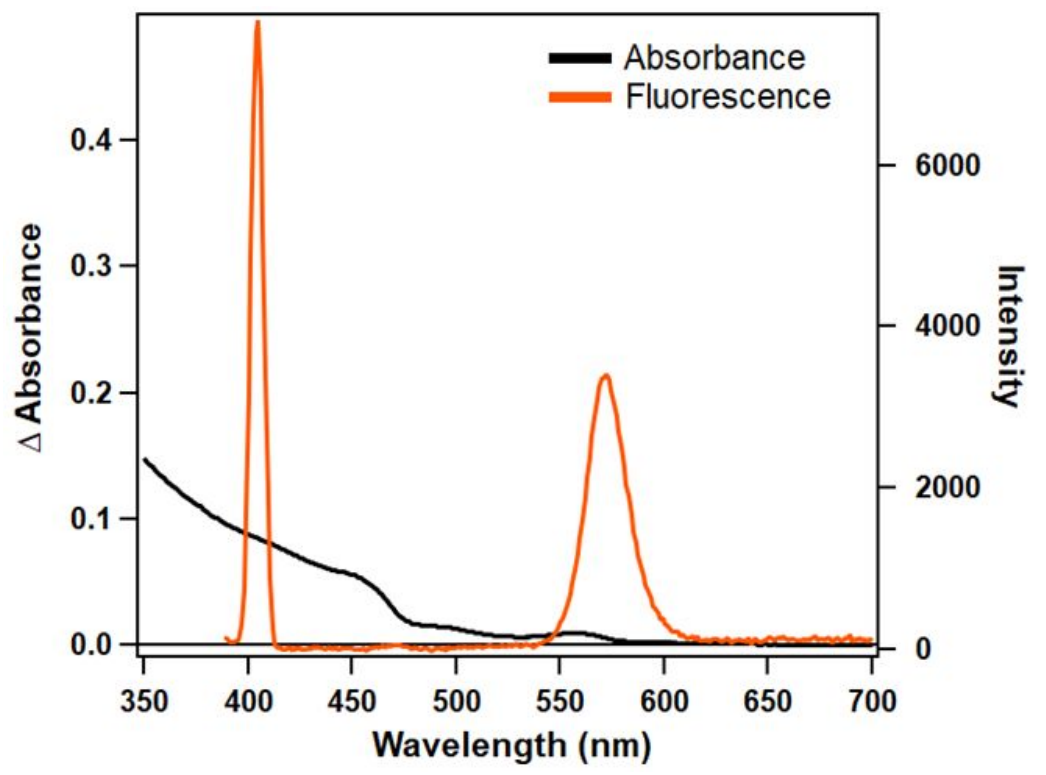

Figure S2. Absorbance and fluorescence spectra of nanorods. UV-Vis spectra were recorded on a Perkin Elmer Lambda 35 Spectrometer suspended in water and the fluorescence spectra were recorded on a Horiba Scientific Fluorometer suspended in chloroform, with an excitation wavelength of $405 \mathrm{~nm}$. 


\section{Section 2. Quantum Efficiency of Photo-driven Mediator Reduction}

\section{A) Representative UV-Vis Spectra of Mediator Radicals: DQ03, DQ53 and DQ43}
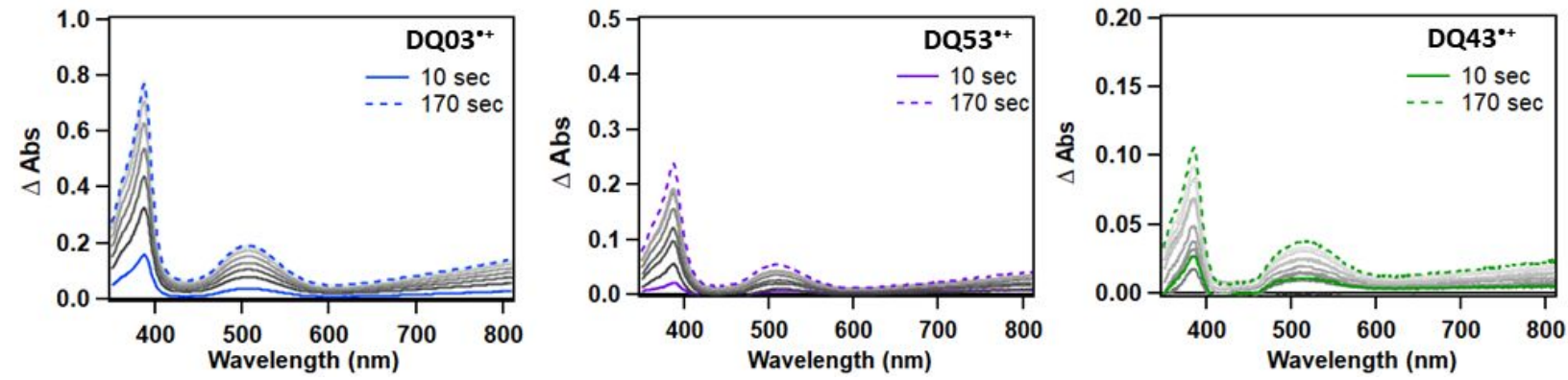

Figure S3. Radical spectra were obtained by illuminating a sample containing NRs, mediator, and sacrificial electron donor with $405 \mathrm{~nm}$ light (4.4 mW, purchased from Thor labs) which was housed in a quartz cuvette. Spectra were recorded on a fiber optically coupled uv-vis absorbance spectrometer.

B) Table S1: pH Dependence of QE (\%) of Photo-Driven Mediator Reduction

\begin{tabular}{|c|c|c|c|}
\hline & DQ03 & DQ53 & DQ43 \\
\hline pH 6.5 & $68 \pm 4$ & $31 \pm 5$ & $7 \pm 2$ \\
\hline pH 8.5 & $100 \pm 1$ & $100 \pm 15$ & $36 \pm 12$ \\
\hline pH 10.5 & $95 \pm 6$ & $95 \pm 8$ & $64 \pm 2$ \\
\hline
\end{tabular}

C) Table S2: Mediator Concentration Dependence of QE (\%) of Photo-Driven Mediator Reduction

\begin{tabular}{|c|c|c|c|}
\hline & DQ03 & DQ53 & DQ43 \\
\hline $\mathbf{1 ~} \mathbf{~ M M}$ & $78 \pm 11$ & $38 \pm 5$ & $19 \pm 3$ \\
\hline $\mathbf{5} \mathbf{~ m M}$ & $84 \pm 9$ & $65 \pm 4$ & $27 \pm 2$ \\
\hline $10 \mathbf{~ m M}$ & $94 \pm 1$ & $66 \pm 7$ & $26 \pm 5$ \\
\hline $\mathbf{3 0} \mathbf{~ m M}$ & $96 \pm 5$ & $70 \pm 1$ & $40 \pm 5$ \\
\hline
\end{tabular}




\section{D) QE Calculation}

$$
\begin{gathered}
\mathrm{QE}=\frac{\text { Mols of Mediator Radical }}{\text { Mols of Photons }} \\
\mathrm{QE}=\frac{\text { mols of mediator radical }}{\left.(\text { photons per sec }) *\left(1-10^{(\mathrm{NR} \text { abs } @ 405 \mathrm{~nm})}\right)\right) *(\mathrm{sec})}
\end{gathered}
$$

QE was calculated using the equation above. Moles of mediator radical generated divided by the moles of photons absorbed. 


\section{Section 3. Steady State Solution Potential During H2 Production(left) and H2 Production in the absence of Enzyme (right)}
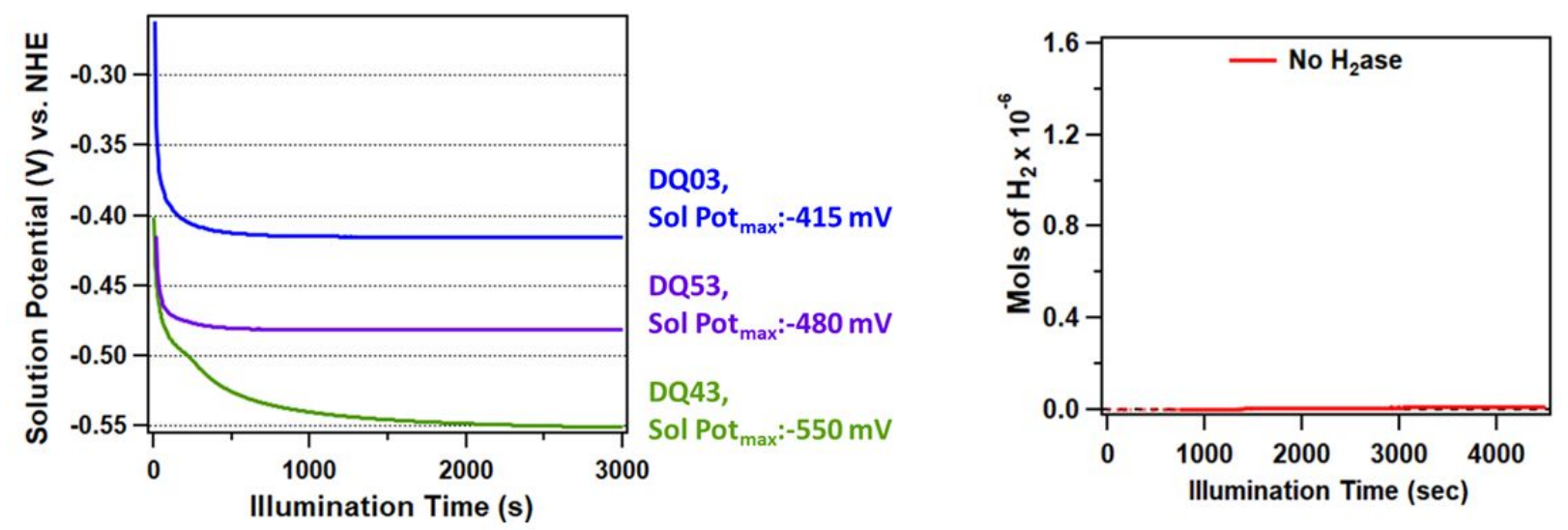

Figure S4. Spectra during $\mathrm{H}_{2}$ production assays were recorded on a fiber optically coupled Ocean Optics UV-Visible absorbance spectrometer. The solution potential was determined by quantifying the radical concentration in solution from uv-vis absorbance spectra and calculating the real time solution potential using the Nernst Equation. Solution Potential Maximum (Sol Pot $\mathrm{max}_{\text {) }}$ has been calculated at $2500 \mathrm{sec}$. The figure above (left) is a plot of solution potential ( $\mathrm{mV}$ ) vs Illumination Time (sec).

Plot of mol of $\mathrm{H}_{2}$ generated when only $\mathrm{CdSe} / \mathrm{CdS} \mathrm{NRs}$ and mediator (DQ03) are present (right). The $\mathrm{H}_{2}$ produced was measured by a pressure sensor inserted into the cap of the cuvette. Similar results were obtained with mediators, DQ43 and DQ53. 
Section 4. Fit Coefficients for TCSPC Measurements

\begin{tabular}{|c|c|c|c|c|c|c|c|}
\hline & $\mathbf{A}_{\mathbf{1}}$ & $\boldsymbol{\tau}_{\mathbf{1}}$ (ns) & $\mathbf{A}_{\mathbf{2}}$ & $\boldsymbol{\tau}_{\mathbf{2}}$ (ns) & $\mathbf{A}_{\mathbf{3}}$ & $\boldsymbol{\tau}_{\mathbf{3}}$ (ns) & $\mathbf{A W L}$ \\
\hline pH 6.5 & 2.717 & 8.758 & 4.643 & 2.736 & 4.55 & 0.455 & $\mathbf{3 . 2 4} \mathbf{n s}$ \\
\hline pH 8.5 & 0.967 & 6.64 & 9.51 & 0.315 & 4.505 & 2.041 & $\mathbf{1 . 2 4} \mathbf{n s}$ \\
\hline pH 10.5 & 0.526 & 4.8 & 17.7 & 0.187 & 3.27 & 1.25 & $\mathbf{0 . 4 6} \mathbf{n s}$ \\
\hline
\end{tabular}

Table S3. Time correlated single photon counting measurements were recorded on a Nikon confocal scanning laser microscope interfaced with Picoquant TCSPC electronics.

Samples were prepared anaerobically in a well plate and sealed with VWR well plate sealing film. 


\section{Section 5. Data Analysis Procedure for Transient Visible Measurements}

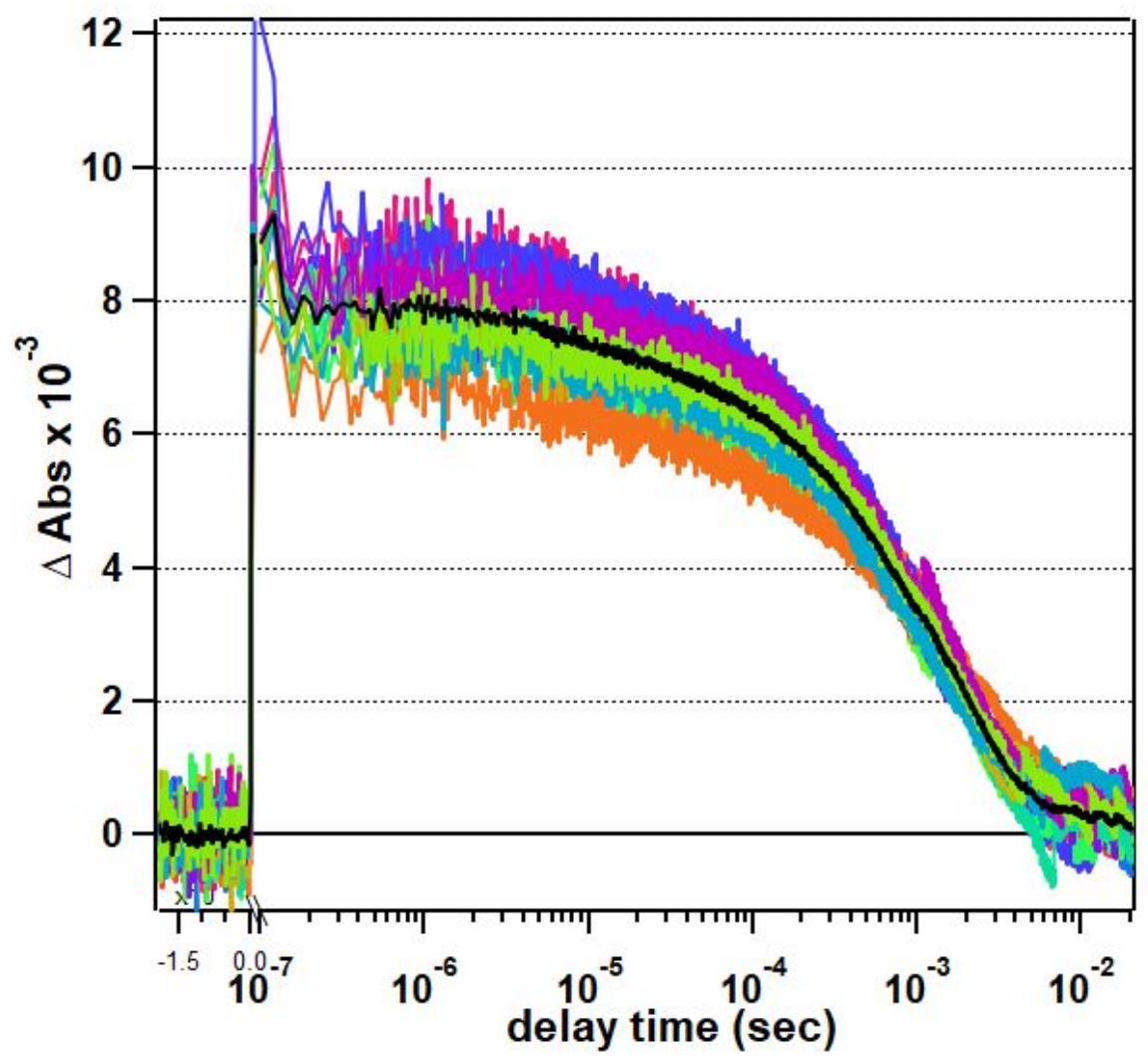

Figure S6. Pictured above is representative raw data from transient absorbance potential jump measurements from monitoring at $785 \mathrm{~nm}$. Multicolored traces represent individual shots and the black trace is the average of these traces and is used for solution potential calculations. 


\section{Section 6. Fitting Procedure for Time Resolved Visible Data}

\section{A) $1 \mathrm{mM}$ CrHydA1 Sample TR Visible Data}
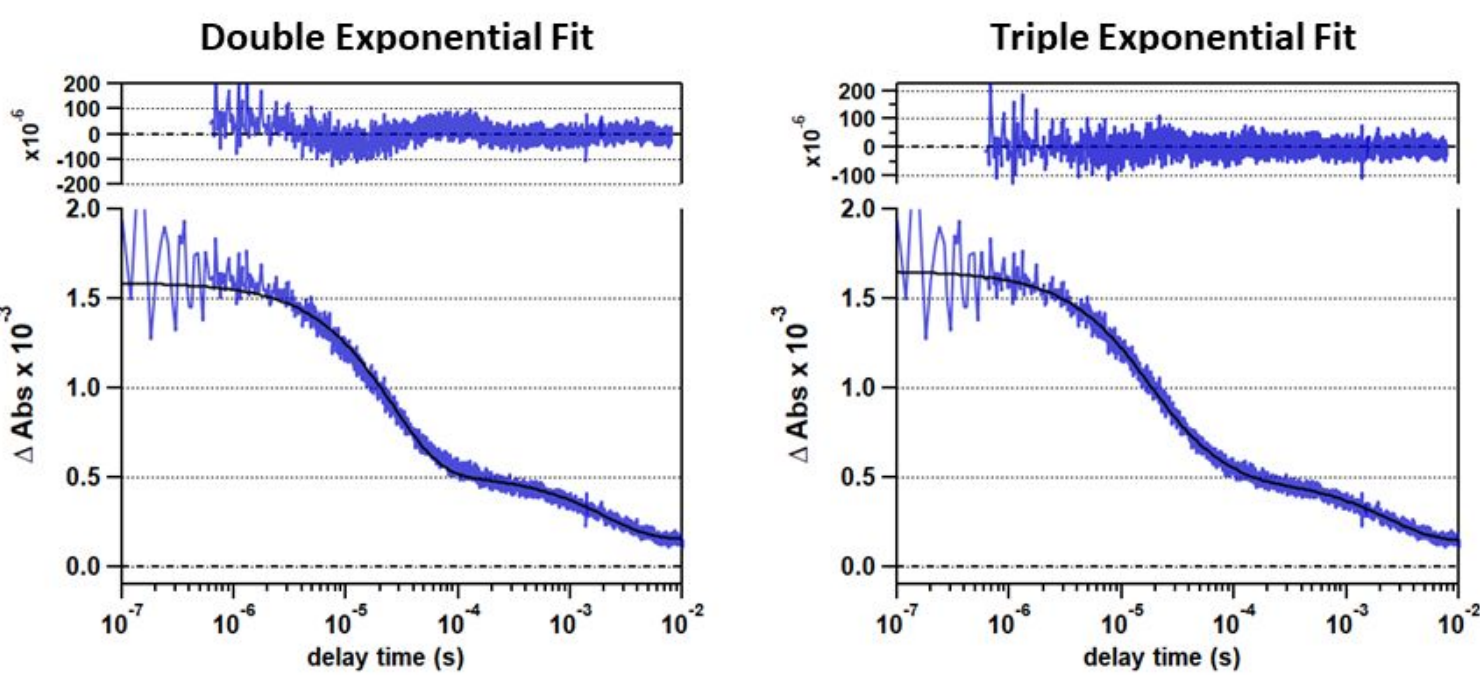

\begin{tabular}{|c|c|c|c|c|c|c|c|}
\hline & $\mathbf{A}_{\mathbf{1}}$ & $\boldsymbol{\tau}_{\mathbf{1}}$ & $\mathbf{A}_{\mathbf{2}}$ & $\boldsymbol{\tau}_{\mathbf{2}}$ & $\mathbf{A}_{\mathbf{3}}$ & $\boldsymbol{\tau}_{\mathbf{3}}$ & $\mathbf{C h i}^{\mathbf{2}}$ \\
\hline Double & 0.0011 & $25.7 \mu \mathrm{s}$ & 0.00036 & 0.0023 & & & $6.25 \mathrm{E}-6$ \\
\hline Triple & 0.00075 & $14.7 \mu \mathrm{s}$ & 0.00042 & $57.5 \mu \mathrm{s}$ & 0.00035 & $2.3 \mathrm{~ms}$ & $4.77 \mathrm{E}-6$ \\
\hline
\end{tabular}

*highlighted row denotes values used for analysis

\section{B) 4 mM CrHydA1 Sample TR Visible data}
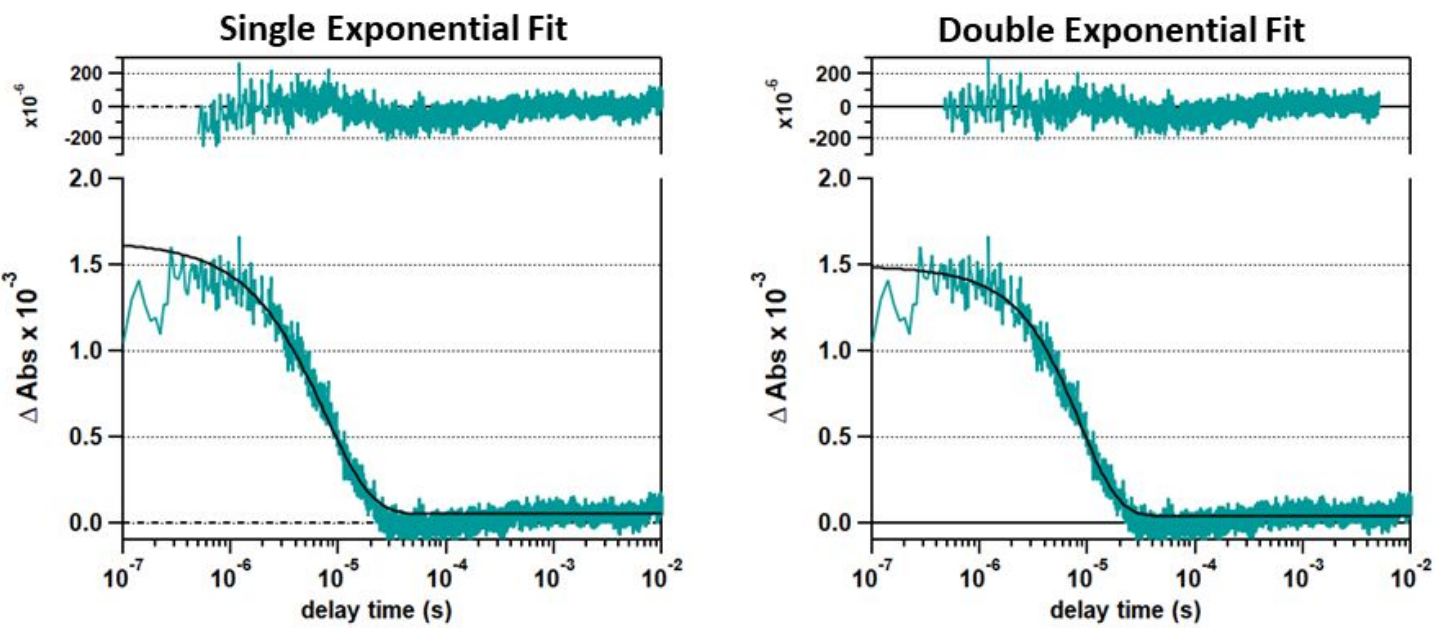

\begin{tabular}{|c|c|c|c|c|c|}
\hline & $\mathbf{A}_{\mathbf{1}}$ & $\boldsymbol{\tau}_{\mathbf{1}}$ & $\mathbf{A}_{\mathbf{2}}$ & $\boldsymbol{\tau}_{\mathbf{2}}$ & $\mathbf{C h i}^{\mathbf{2}}$ \\
\hline Single & 0.00158 & $7.5 \mu \mathrm{s}$ & & & $2.75 \mathrm{E}-5$ \\
\hline Double & 0.0031 & $5.8 \mu \mathrm{s}$ & -.0016 & $3.8 \mu \mathrm{s}$ & $1.54 \mathrm{E}-5$ \\
\hline
\end{tabular}

*highlighted row denotes values used for analysis 


\section{Section 7. Calculation of Laser-Induced Potential Jump}

From monitoring the radical band at $785 \mathrm{~nm}$, we can obtain the concentration of radical generated $(\triangle \mathrm{A})$ with each pump of the sample volume using Beer's Law. With the Nernst equation (below) we can calculate the magnitude of the solution potential jump from the concentration of radical generated similar to what has been done previously. ${ }^{1-3}$

Calculation of Mediator ${ }^{\bullet+}$ concentration (DQ03):

$$
\begin{gathered}
A=\varepsilon * c * l \\
0.01=70000 \mathrm{~mol}^{-1} \mathrm{~m}^{-1} * c * 0.00005 \mathrm{~m} \\
\boldsymbol{c}=\mathbf{2 . 9} \mathbf{m M}
\end{gathered}
$$

Where,

$$
\begin{aligned}
& A=\text { absorbance } \\
& \varepsilon=\text { molar absorptivity, } M^{-1} \mathrm{~m}^{-1} \\
& c=\text { concentration of mediator } \\
& \text { I= sample pathlength }(50 \mu \mathrm{m})
\end{aligned}
$$

Calculation of $\Delta V(D Q 03)$ :

$$
\begin{gathered}
E=E^{\circ}-\frac{R T}{F n} * \ln \left(\frac{[\text { reduced }]}{\text { [oxidized }]}\right) \\
E=-0.55 \mathrm{~V}-\frac{\left(8.314^{J} / \mathrm{mol} \mathrm{K} * 298 \mathrm{~K}\right)}{\left(96,486^{C} / \mathrm{mol}^{* 1}\right)} * \ln \left(\frac{[2.9 \mathrm{mM}]}{[25 \mathrm{mM}]}\right) \\
\boldsymbol{E}=-\mathbf{0 . 4 9 7} \mathbf{V}
\end{gathered}
$$

Where,

$R=$ gas constant

$T=$ temperature $(K)$

$F=$ Faraday's constant

$E=\Delta V$, magnitude of solution potential jump

$E^{\circ}=$ reduction potential of mediator $(-0.55 \mathrm{~V})$

$n=\#$ of electrons

$[$ reduced $]=$ concentration of reduced mediator

[oxidized $]=$ concentration of oxidized mediator 


\section{Section 8. Summary of Solution Potential Values}

Table S4 summarizing solution potential values (in $\mathrm{mV}$ ) from transient measurements

\begin{tabular}{|c|c|c|c|c|c|c|c|c|c|}
\hline & \multicolumn{3}{|c|}{ DQ03 } & \multicolumn{3}{c|}{ DQ53 } & \multicolumn{3}{c|}{ DQ43 } \\
\hline $\begin{array}{c}\text { Pump Power } \\
\text { (Watts) }\end{array}$ & $\mathrm{pH} 6.5$ & $\mathrm{pH} 7.5$ & $\mathrm{pH} 8.5$ & $\mathrm{pH} 6.5$ & $\mathrm{pH} 7.5$ & $\mathrm{pH} 8.5$ & $\mathrm{pH} 6.5$ & $\mathrm{pH} 7.5$ & $\mathrm{pH} 8.5$ \\
\hline $\mathbf{0 . 0 5}$ & -395 & -406 & -428 & -442 & -466 & -507 & -490 & -536 & -544 \\
\hline $\mathbf{0 . 1 0}$ & -412 & -427 & -438 & -460 & -486 & -510 & -498 & -534 & -557 \\
\hline $\mathbf{0 . 1 5}$ & -428 & -436 & -446 & -472 & -493 & -524 & -507 & -541 & -569 \\
\hline $\mathbf{0 . 2 0}$ & -438 & -441 & -448 & -486 & -497 & -526 & -524 & -545 & -578 \\
\hline $\mathbf{0 . 3 5}$ & -444 & -452 & -458 & -513 & -504 & -532 & -541 & -547 & -583 \\
\hline $\mathbf{0 . 5 0}$ & & -457 & -473 & & -512 & -548 & & -554 & -592 \\
\hline
\end{tabular}

*Solution potential for transient measurements were calculated from $\Delta \mathrm{A}$ at $1 \mu \mathrm{s}$. 
Section 9. Fit Coefficients for Time-Resolved IR Measurements

\begin{tabular}{|c|c|c|c|c|c|}
\hline \multicolumn{7}{|c|}{ TRIR Multi Exponential Fit Coefficients } \\
\hline Protein Concentration & Probe Frequency & $\mathbf{A}_{\mathbf{1}}$ & $\boldsymbol{\tau}_{\mathbf{1}}$ & $\mathbf{A}_{\mathbf{2}}$ & $\boldsymbol{\tau}_{\mathbf{2}}$ \\
\hline $1 \mathrm{mM}$ Sample & $1941 \mathrm{~cm}^{-1}\left(\mathrm{H}_{\mathrm{ox}}\right)$ & 0.0011 & $28 \mu \mathrm{s}$ & 0.0012 & $90 \mu \mathrm{s}$ \\
\hline $4 \mathrm{mM}$ Sample & $1941 \mathrm{~cm}^{-1}\left(\mathrm{H}_{\mathrm{ox}}\right)$ & 0.003 & $7 \mu \mathrm{s}$ & 0.0027 & $35 \mu \mathrm{s}$ \\
\hline $1 \mathrm{mM}$ Sample & $1934 \mathrm{~cm}^{-1}\left(\mathrm{H}_{\mathrm{red}}\right)$ & -0.0004 & $20 \mu \mathrm{s}$ & -0.001 & $94 \mu \mathrm{s}$ \\
\hline $4 \mathrm{mM}$ Sample & $1934 \mathrm{~cm}^{-1}\left(\mathrm{H}_{\mathrm{red}}\right)$ & -0.001 & $5 \mu \mathrm{s}$ & -0.0017 & $24 \mu \mathrm{s}$ \\
\hline $1 \mathrm{mM}$ Sample & $1891 \mathrm{~cm}^{-1}\left(\mathrm{H}_{\mathrm{red}} \mathrm{H}^{+}\right)$ & -0.0002 & $29 \mu \mathrm{s}$ & 0.0003 & $1.1 \mathrm{~ms}$ \\
\hline $4 \mathrm{mM}$ Sample & $1891 \mathrm{~cm}^{-1}\left(\mathrm{H}_{\mathrm{red}} \mathrm{H}^{+}\right)$ & -0.0019 & $8.5 \mu \mathrm{s}$ & 0.0016 & $0.4 \mathrm{~ms}$ \\
\hline $1 \mathrm{mM}$ Sample & $1882 \mathrm{~cm}^{-1}\left(\mathrm{H}_{\text {sred }} \mathrm{H}^{+}\right)$ & -0.00015 & $19 \mu \mathrm{s}$ & 0.00012 & $460 \mu \mathrm{s}$ \\
\hline $4 \mathrm{mM}$ Sample & $1882 \mathrm{~cm}^{-1}\left(\mathrm{H}_{\text {sred }} \mathrm{H}^{+}\right)$ & -0.00041 & $8 \mu \mathrm{s}$ & 0.00021 & $130 \mu \mathrm{s}$ \\
\hline
\end{tabular}

Section 10. Fit coefficients for Time Resolved Visible Measurements

\begin{tabular}{|c|c|c|c|c|c|c|}
\hline \multicolumn{7}{|c|}{ TRVis Multi Exponential Fit Coefficients } \\
\hline & $\mathbf{A}_{\mathbf{1}}$ & $\boldsymbol{\tau}_{\mathbf{1}}$ & $\mathbf{A}_{\mathbf{2}}$ & $\boldsymbol{\tau}_{\mathbf{2}}$ & $\mathbf{A}_{\mathbf{3}}$ & $\boldsymbol{\tau}_{\mathbf{3}}$ \\
\hline $1 \mathrm{mM}$ Sample & 0.0007 & $15 \mu \mathrm{s}$ & 0.0004 & $60 \mu \mathrm{s}$ & 0.00035 & $2.2 \mathrm{~ms}$ \\
\hline $4 \mathrm{mM}$ Sample & 0.0011 & $8 \mu \mathrm{s}$ & & & & \\
\hline
\end{tabular}




\section{Section 11. Proposed Catalytic Cycle for [FeFe] Hydrogenase}
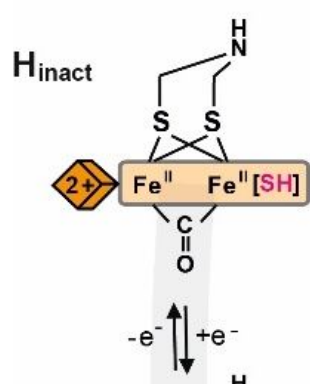

$H_{\text {trans }}$
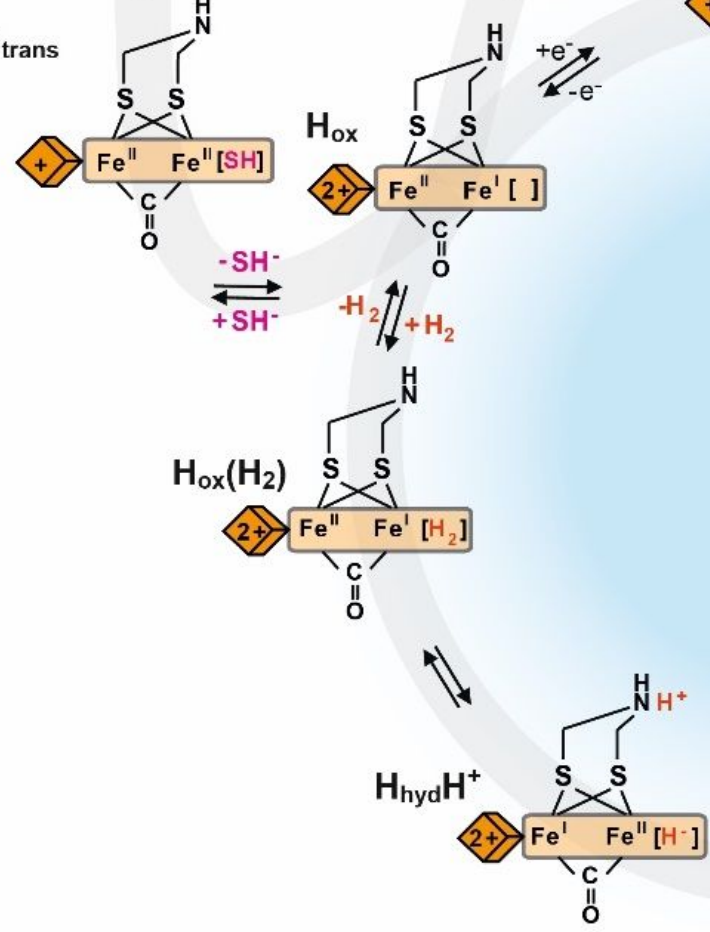

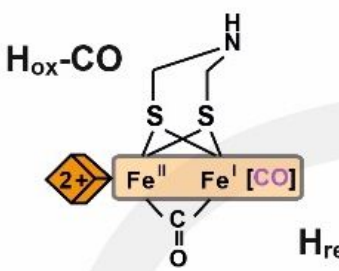

$+\mathrm{co} / 4-\mathrm{Co}$
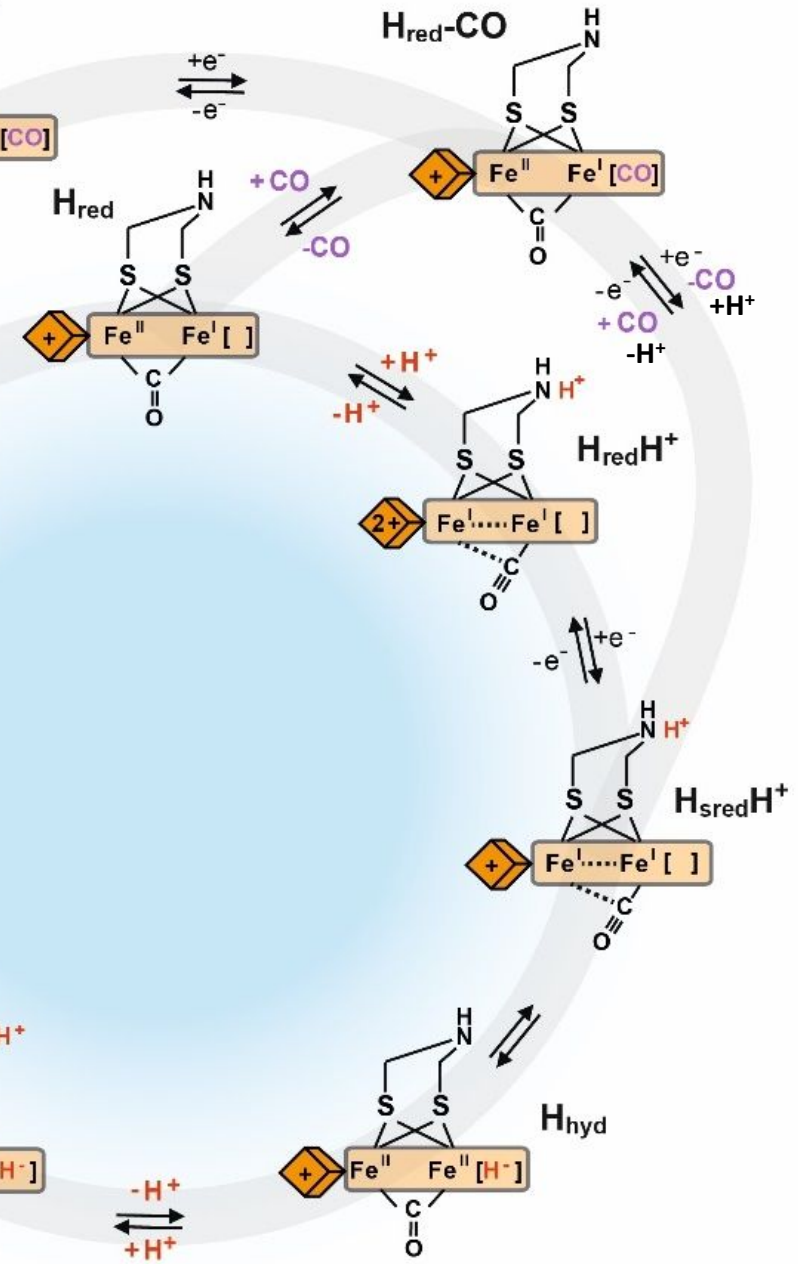

Figure S7. Proposed catalytic cycle for [FeFe] hydrogenase including off pathway intermediate states, based on previously published results. ${ }^{1,4-8}$ 


\section{Section 12. References}

1. Sanchez, M. L. K.; Sommer, C.; Reijerse, E.; Birrell, J. A.; Lubitz, W.; Dyer, R. B., Investigating the Kinetic Competency of CrHydA1 [FeFe] Hydrogenase Intermediate States via Time-Resolved Infrared Spectroscopy. J. Am. Chem. Soc. 2019, 16064-16070.

2. Sanchez, M. L. K.; Wu, C.-H.; Adams, M. W. W.; Dyer, R. B., Optimizing electron transfer from CdSe QDs to hydrogenase for photocatalytic H2 production. Chem. Comm. 2019, 5579-5582.

3. Chica, B.; Wu, C.-H.; Liu, Y.; Adams, M. W. W.; Lian, T.; Dyer, R. B., Balancing electron transfer rate and driving force for efficient photocatalytic hydrogen production in $\mathrm{CdSe} / \mathrm{CdS}$ nanorod-[NiFe] hydrogenase assemblies. Energy Environ. Sci. 2017, 2245-2255.

4. Adamska, A.; Silakov, A.; Lambertz, C.; Rudiger, O.; Happe, T.; Reijerse, E.; Lubitz, W., Identification and characterization of the "super-reduced" state of the H-cluster in [FeFe] hydrogenase: a new building block for the catalytic cycle? Angew. Chem. Int. Ed. 2012, 11458-62.

5. Adamska-Venkatesh, A.; Krawietz, D.; Siebel, J.; Weber, K.; Happe, T.; Reijerse, E.; Lubitz, W., New Redox States Observed in [FeFe] Hydrogenases Reveal Redox Coupling Within the H-Cluster. J. Am. Chem. Soc. 2014, 11339-11346.

6. Sommer, C.; Adamska-Venkatesh, A.; Pawlak, K.; Birrell, J. A.; Rudiger, O.; Reijerse, E. J.; Lubitz, W., Proton Coupled Electronic Rearrangement within the H-Cluster as an Essential Step in the Catalytic Cycle of [FeFe] Hydrogenases. J. Am. Chem. Soc. 2017, 1440-1443.

7. Rodríguez-Maciá, P.; Reijerse, E. J.; van Gastel, M.; DeBeer, S.; Lubitz, W.; Rüdiger, O.; Birrell, J. A., Sulfide Protects [FeFe] Hydrogenases From O2. J. Am. Chem. Soc. 2018, 9346-9350.

8. Birrell, J. A.; Pelmenschikov, V.; Mishra, N.; Wang, H.; Yoda, Y.; Tamasaku, K.; Rauchfuss, T. B.; Cramer, S. P.; Lubitz, W.; DeBeer, S., Spectroscopic and Computational Evidence that [FeFe] Hydrogenases Operate Exclusively with CO-Bridged Intermediates. J. Am. Chem. Soc. 2020, 222-232. 